\title{
Charge state dynamics and optically detected electron spin resonance contrast of shallow nitrogen-vacancy centers in diamond
}

\author{
Zhiyang Yuan, Mattias Fitzpatrick $\odot$, Lila V. H. Rodgers, Sorawis Sangtawesin $\odot,{ }^{*}$ \\ Srikanth Srinivasan $\odot,{ }^{\dagger}$ and Nathalie P. de Leon ${ }^{\ddagger}$ \\ Department of Electrical Engineering, Princeton University, Princeton, New Jersey 08544, USA
}

(Received 3 May 2020; revised 1 July 2020; accepted 3 August 2020; published 18 August 2020)

\begin{abstract}
Nitrogen-vacancy (NV) centers in diamond can be used for nanoscale sensing with atomic resolution and sensitivity; however, it has been observed that their properties degrade as they approach the diamond surface. Here we report that in addition to degraded spin coherence, NV centers within nanometers of the surface can also exhibit decreased fluorescence contrast for optically detected electron spin resonance (OD-ESR). We demonstrate that this decreased OD-ESR contrast arises from charge state dynamics of the NV center, and that it is strongly surface-dependent, indicating that surface engineering will be critical for nanoscale sensing applications based on color centers in diamond.
\end{abstract}

DOI: 10.1103/PhysRevResearch.2.033263

\section{INTRODUCTION}

Nitrogen-vacancy (NV) centers in diamond are actively explored for a number of applications in quantum information processing and sensing because they exhibit long spin coherence times at room temperature, and their spin states can be optically initialized and read out with off-resonant excitation [1-6]. In order to achieve strong interactions with materials and molecules that are external to the diamond, NV centers must be placed close to the diamond surface. It has been well established that the diamond surface can host contaminants, magnetic defects, and electronic defects that give rise to noise, leading to short spin coherence times [7-16], and recent work has shown that careful preparation of the diamond surface can mitigate this noise, leading to extended spin coherence times $[6,7,9]$. Most NV center applications are based on the negative charge state of $\mathrm{NV}$ centers, $\mathrm{NV}^{-}$, and it is also known that $\mathrm{NV}$ centers can exist in the neutral charge state, $\mathrm{NV}^{0}$. It has been recently shown that both the steady-state charge populations under illumination and the equilibrium charge state in the dark of shallow NV centers can vary significantly from bulk properties [17-20]. Furthermore, despite the strong motivation to use NV centers as close to the surface as possible for scanning probe measurements, we note that essentially all reported experiments utilize NV centers around or deeper

\footnotetext{
*Present address: School of Physics, Suranaree University of Technology, Nakhon Ratchasima 30000, Thailand.

†resent address: IBM T. J. Watson Research Center, Yorktown Heights, NY 10598, USA.

${ }^{\ddagger}$ npdeleon@princeton.edu

Published by the American Physical Society under the terms of the Creative Commons Attribution 4.0 International license. Further distribution of this work must maintain attribution to the author(s) and the published article's title, journal citation, and DOI.
}

than $10 \mathrm{~nm}$ from the surface [21-26], indicating that shallower NV centers likely have an unstable charge state. Finally, as we have previously reported [7], high-temperature annealing can result in surfaces with no observable NV centers, which are then subsequently recovered after annealing in an oxygen atmosphere. Since annealing in oxygen reduces the density of electronic traps at the surface, this behavior is likely explained by charge state instability induced by nearby electronic traps. Previous work has shown that charge state initialization can influence spin readout [27]; however, a detailed understanding of how these charge state dynamics can affect schemes for nanoscale sensing and its dependence on sample surface has not yet been established.

Here we demonstrate that the diamond surface can strongly affect both the steady-state charge state distribution and the charge state dynamics of NV centers within nanometers of the diamond surface, and that these charge state properties can significantly degrade optically detected electron spin resonance (OD-ESR) contrast. In order to quantify the impact of charge state dynamics on OD-ESR contrast, we focus on two diamond samples (samples A and F) that contain shallow NV centers introduced by ion implantation, which exhibit distinctly different OD-ESR contrast and charge state behavior. We find that sample A exhibits higher contrast, greater charge state stability in the dark, lower $\mathrm{NV}^{0}$ population under green $(532 \mathrm{~nm})$ illumination, and slower ionization and recombination dynamics than sample F. More specifically, we find that sample $\mathrm{F}$ exhibits charge state conversion rates that are comparable to the internal spin-dependent dynamics of the NV center, thus leading to decreased OD-ESR contrast. Finally, we measure time-resolved, spin-dependent photoluminescence (PL) and compare to a coupled rate equation model that incorporates charge conversion rates to quantitatively understand the impact of charge dynamics on OD-ESR contrast.

A cartoon schematic of the interactions between shallow NV centers and charge traps is depicted in Fig. 1(a). The surface can host defects that act as charge traps, and tunneling 
(a)

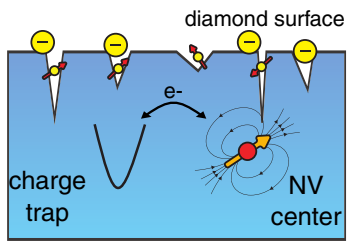

(c)

(b)
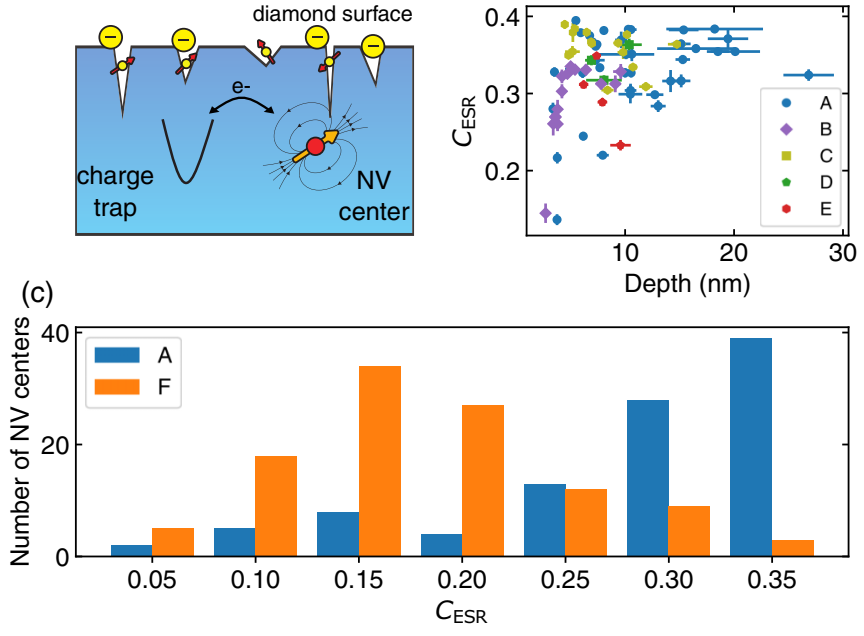

FIG. 1. Variation in OD-ESR contrast for shallow NV centers. (a) Cartoon depicting the interaction between shallow NV centers and defects at the diamond surface. Defects can act as charge traps, leading to changes in ionization and recombination kinetics for nearby NV centers. (b) OD-ESR contrast, $C_{\mathrm{ESR}}$, as a function of depth for NV centers across five diamond samples. A large spread and an average decrease in contrast are apparent within around $10 \mathrm{~nm}$ of the surface. (c) Histograms of $C_{\mathrm{ESR}}$ from the two selected samples, A (blue bars) and F (orange bars). $99 \mathrm{NV}$ centers in sample A and 108 $\mathrm{NV}$ centers in sample F were measured under the same experimental conditions including microwave pulse power and green laser power. An external magnetic field of approximately $27 \mathrm{G}$ was aligned to the NV axis.

from NV centers to these charge traps gives rise to charge state instability, leading to a decrease in OD-ESR contrast of shallow NV centers [17].

Following Ref. [28], we define OD-ESR contrast as

$$
C_{\mathrm{ESR}}=\frac{\alpha_{0}-\alpha_{ \pm 1}}{\alpha_{0}},
$$

where $\alpha_{i}$ is the average number of photons collected in the readout pulse when the $\mathrm{NV}^{-}$spin state is initialized to the $m_{s}=i$ state. Figure 1 (b) shows $C_{\mathrm{ESR}}$ plotted for many NV centers as a function of depth across five samples, where depth is measured by detecting the proton NMR signal arising from the microscope immersion oil [29]. NV centers within $10 \mathrm{~nm}$ of the surface display a wider distribution in $C_{\mathrm{ESR}}$.

\section{SAMPLES}

In order to investigate the origin of the lower OD-ESR contrast, we identify two samples that have undergone nominally similar surface processing (see Appendix A), but exhibit markedly different distributions in $C_{\mathrm{ESR}}$, samples $\mathrm{A}$ and $\mathrm{F}$ [Fig. 1(c)]. Most NV centers in sample F exhibit $C_{\text {ESR }}$ below 0.3 , as well as short coherence times $\left(T_{2}<4 \mu \mathrm{s}\right)$, precluding many NV characterization measurements, such as single-shot charge state readout [30] and using proton NMR to measure depth. We use this comparison to quantify the impact of charge state conversion on $C_{\mathrm{ESR}}$.

Both samples were prepared using nitrogen ion implantation followed by thermal annealing. Sample A was implanted with a nitrogen ion energy of $3 \mathrm{keV}$ while sample $\mathrm{F}$ was implanted with an energy of $1.5 \mathrm{keV}$. Despite undergoing nominally similar surface processing, sample F contains persistent boron contamination comprising approximately $4 \%$ of a surface monolayer as measured by x-ray photoelectron spectroscopy (see Appendix A), likely arising from contamination in the furnace during vacuum thermal annealing. We note that although sample $\mathrm{F}$ is expected to have a shallower distribution of NV centers because of its lower implantation energy, the distribution of $C_{\mathrm{ESR}}$ is much lower than another sample that was prepared with the same ion implantation energy that does not exhibit surface contamination, sample B (also see Appendix A).

\section{EXPERIMENTAL RESULTS}

\section{A. Equilibrium charge state properties}

To understand the origin of the lower OD-ESR contrast in sample F, we examine the steady-state charge distributions in both samples. First we measure the PL spectrum under green illumination. We observe a significantly higher fraction of emission between the zero phonon line (ZPL) of $\mathrm{NV}^{0}$ $(575 \mathrm{~nm})$ and the $\mathrm{ZPL}$ of $\mathrm{NV}^{-}(637 \mathrm{~nm})$ in sample $\mathrm{F}$, as well as a peak at $575 \mathrm{~nm}$ [Fig. 2(a)], indicating a higher steady-state population of $\mathrm{NV}^{0}$ [31]. This higher $\mathrm{NV}^{0}$ steady-state population contributes to background fluorescence, decreasing the OD-ESR contrast proportionally.

\section{B. Charge conversion in the dark}

Recent work has shown that shallow NV centers can exhibit spontaneous conversion to $\mathrm{NV}^{0}$ without illumination [17]. Here, we perform a similar measurement on several NV centers in both samples. First, the NV center charge state is initialized using a green pulse of sufficient length $(5 \mathrm{~ms}$ at $510 \mu \mathrm{W})$ to achieve a steady-state distribution. Then, after a variable time interval in the dark, we measure PL during an orange $(590 \mathrm{~nm})$ pulse, which preferentially excites $\mathrm{NV}^{-}$, allowing for charge state readout. Normalizing to the PL at the shortest dark time interval, we observe that NV centers in sample $\mathrm{F}$ exhibit a decay in the $\mathrm{NV}^{-}$population over timescales between $11 \mathrm{~ms}$ and $300 \mathrm{~ms}$ to less than half of the initial value, while a decay of less than $25 \%$ is observed in sample A for dark times up to $1 \mathrm{~s}$ [Fig. 2(b)]. In sample A, we also perform the inverse measurement by initializing $\mathrm{NV}$ centers into $\mathrm{NV}^{0}$ using an orange pulse $(5 \mathrm{~ms}$ at $18 \mu \mathrm{W}$, after a green pulse as Fig. 2(b) for consistency [19]) to look for evidence of spontaneous conversion to $\mathrm{NV}^{-}$. We observe no change in the $\mathrm{NV}^{-}$population out to $1 \mathrm{~s}$ [Fig. 2(c)], indicating that spontaneous charge conversion is very slow in this sample, regardless of the initial charge state population. This is consistent with previous hypotheses that spontaneous charge conversion is mediated by the availability of nearby electron traps at or near the surface [Fig. 1(a)] that strongly modify charge state kinetics [17]. 
(a)
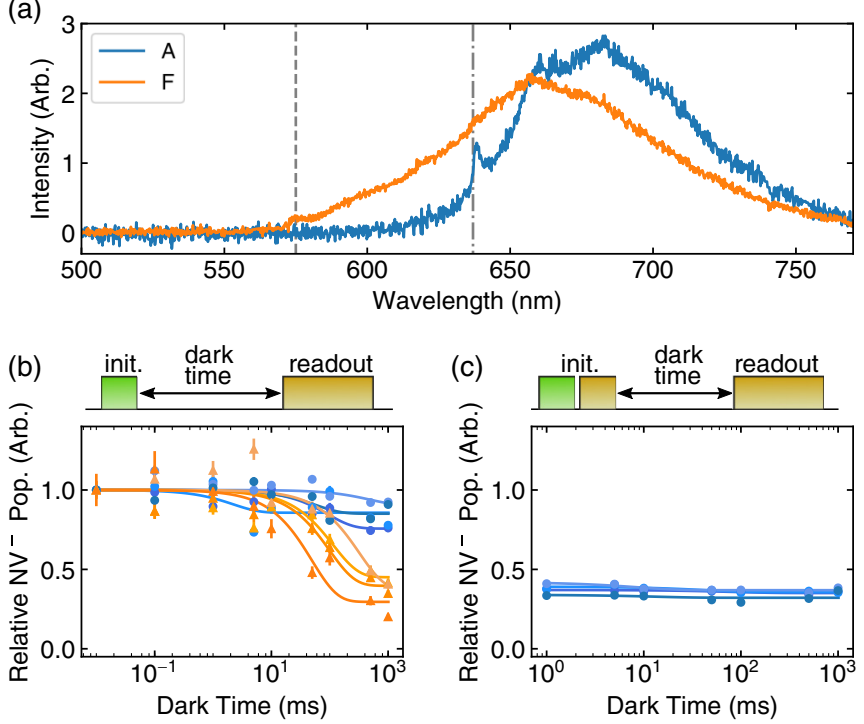

FIG. 2. Steady-state charge distributions. (a) PL spectrum under green illumination for representative NV centers in sample A (blue trace) and sample F (orange trace). The higher level of PL between $575 \mathrm{~nm}$ (dashed line) and $637 \mathrm{~nm}$ (dash-dotted line) in sample F indicates a higher steady-state population in $\mathrm{NV}^{0}$. (b) Charge state conversion in the dark. NV centers are initialized into the negative charge state by a green pulse, and the charge state is read out using a charge-state-selective orange pulse with variable delay time. The PL is normalized to the value for the shortest dark time interval. Each $\mathrm{NV}$ center in sample $\mathrm{F}$ (orange triangles) exhibits a decay in the relative $\mathrm{NV}^{-}$population with a time constant in the range of 11-300 $\mathrm{ms}$, while NV centers in sample A (blue circles) exhibit much less decay out to $1 \mathrm{~s}$. Solid lines are fits to the data. (c) Stability of $\mathrm{NV}^{0}$ in the dark in sample A. NV centers are initialized into $\mathrm{NV}^{0}$ using a green initialization pulse followed by an orange pulse, and then read out with another orange pulse after a variable delay time. The PL is normalized to the value after the green pulse. No change in the population is observed out to $1 \mathrm{~s}$, indicating that recombination kinetics are also slow in sample A.

\section{Charge conversion under illumination}

Now we turn to the charge state conversion dynamics under illumination for both samples. The rate of change of the charge populations can be expressed in the following simplified model [30]:

$$
\begin{aligned}
& \frac{d \rho_{-}}{d t}=-r_{\text {ion }} \rho_{-}+r_{\text {rec }} \rho_{0}, \\
& \frac{d \rho_{0}}{d t}=r_{\text {ion }} \rho_{-}-r_{\text {rec }} \rho_{0},
\end{aligned}
$$

where $\rho_{-}$is the $\mathrm{NV}^{-}$population, $\rho_{0}$ is the $\mathrm{NV}^{0}$ population, and $r_{\text {ion }}$ and $r_{\text {rec }}$ are ionization and recombination rates, respectively. Since the total population is conserved $\left(\rho_{-}+\rho_{0}=\right.$ $1)$, we can express the time dependence of the population in terms of a total charge conversion rate, $\rho_{-, 0}(t) \propto e^{-r_{\text {tot }} t}$, where $r_{\text {tot }}=r_{\text {ion }}+r_{\text {rec }}$.

We measure charge conversion rates under green illumination by using a fixed orange pulse to initialize the $\mathrm{NV}$ centers primarily into $\mathrm{NV}^{0}$. Then under green illumination, the population shifts to $\mathrm{NV}^{-}$, and we fit the time-resolved
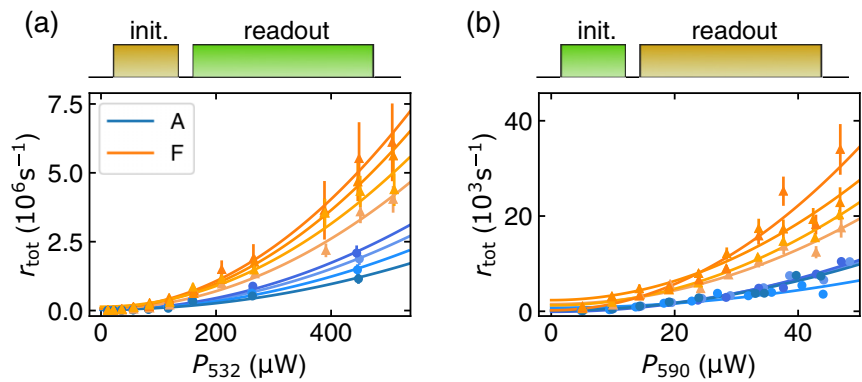

FIG. 3. Charge state dynamics for NV centers in sample A (blue circles) and sample $\mathrm{F}$ (orange triangles). In both plots, points are experimental data and lines are fitted using a quadratic function. (a) Power dependence of the charge state conversion rates under green illumination. (b) Power dependence of the charge state conversion rates under orange illumination. Laser powers are measured at the back of the objective. Under both illumination conditions, the charge state conversion rates are faster for each NV center in sample F compared to those in sample A.

PL to an exponential to extract $r_{\text {tot }}$. Similarly, we measure conversion rates under orange illumination by first initializing with a green pulse and measuring the overall ionization under orange, extracting $r_{\text {tot }}$ in the same manner. Both data sets are plotted in Fig. 3 for a set of NV centers in samples A and $\mathrm{F}$. We observe a clear quadratic power dependence for NV centers in both samples, consistent with both ionization and recombination being two-photon processes [30] (see Appendix E for a simplified derivation). NV centers in sample $\mathrm{F}$ have higher charge conversion rates under both green and orange illumination. Importantly, when the green power is comparable to the typical NV saturation power in our setup $(\approx 300 \mu \mathrm{W})$, the charge state conversion rate can be faster than $1 \times 10^{6} \mathrm{~s}^{-1}$, comparable to the spin polarization rate for $\mathrm{NV}^{-}$, suggesting that fast ionization and recombination processes can occur during the spin initialization and readout times. Since these processes are not spin conserving [32], this fast charge conversion can lead to degradation of the OD-ESR contrast.

\section{TIME-RESOLVED PHOTOLUMINESCENCE MEASUREMENTS: MODEL AND RESULTS}

To quantify how charge state conversion rates affect ODESR contrast for NV centers under distinctly different surface environments, we measure the time-resolved PL during the green readout pulse and fit the traces with a rate equation model which is visualized in Fig. 4. Example PL traces with initial $\mathrm{NV}^{-}$spin states $m_{s}=0$ (upper traces) and $m_{s}=-1$ (lower traces) for NV centers in both samples are shown in Fig. 5(a). The area between the two sets of curves is proportional to $C_{\mathrm{ESR}}$, and in a typical sensing experiment, the readout conditions would be optimized to maximize this area. We observe that the total area between the curves for sample A is $\sim 3.6$ times the area between the curves for sample F. We fit the data using a rate equation model for $\mathrm{NV}^{-}$spin dynamics [32-35], modifying the model to additionally incorporate $\mathrm{NV}^{0}$ states and charge state conversion rates $\Gamma_{\text {ion }}$ and $\Gamma_{\text {rec }}$. In our model, we use five levels to describe the $\mathrm{NV}^{-}$spin states, 


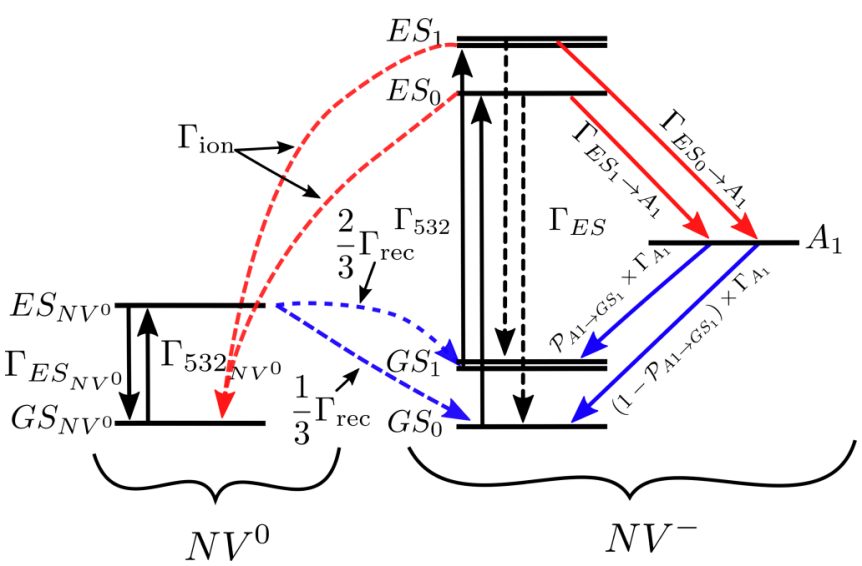

FIG. 4. NV level diagram showing the states considered in our model, which include the ground state triplet $m_{s}=0$ state $\left(\mathrm{GS}_{0}\right)$, the ground state triplet $m_{s}= \pm 1$ states $\left(\mathrm{GS}_{1}\right)$, the excited state triplet $m_{s}=0$ state $\left(\mathrm{ES}_{0}\right)$, the excited state triplet $m_{s}= \pm 1$ states $\left(\mathrm{ES}_{1}\right)$, the singlet state $A_{1}$, and the $\mathrm{NV}^{0}$ ground $\left(\mathrm{GS}_{\mathrm{NV}^{0}}\right)$ and excited $\left(\mathrm{ES}_{\mathrm{NV}^{0}}\right)$ states, along with all corresponding transition rates.

which include two states to describe the ground state triplet $\left(\mathrm{GS}_{0}\right.$ and $\left.\mathrm{GS}_{1}\right)$, two states from the excited state triplet $\left(\mathrm{ES}_{0}\right.$ and $\left.\mathrm{ES}_{1}\right)$, and a single state for the singlet manifold $\left(A_{1}\right)$. We also include ground and excited states for $\mathrm{NV}^{0}\left(\mathrm{GS}_{\mathrm{NV}^{0}}\right.$ and $\mathrm{ES}_{\mathrm{NV}^{0}}$ ). This allows us to describe the system as a state vector

$$
\boldsymbol{\rho}_{\psi}(\boldsymbol{t})=\left[\begin{array}{c}
\rho_{\mathrm{ES}_{1}}(t) \\
\rho_{\mathrm{ES}_{0}}(t) \\
\rho_{A_{1}}(t) \\
\rho_{\mathrm{GS}_{1}}(t) \\
\rho_{\mathrm{GS}_{0}}(t) \\
\rho_{\mathrm{ES}_{\mathrm{Nv}}(t)} \\
\rho_{\mathrm{GS}_{\mathrm{Nv} 0}}(t)
\end{array}\right],
$$

where the entries of the vector $\rho_{\psi}$ describe the probability of finding the system in each of the seven states in our model.

To model the dynamics of the system, we consider the set of transitions that couple all of these spin states, ignoring spinnonconserving transitions (aside from the intersystem crossing and ionization and recombination, which are addressed later). This includes a transition rate $\Gamma_{532}$ between the ground triplet states and the excited triplet states, a transition rate $\Gamma_{\mathrm{ES}}$ between the excited triplet states and the ground triplet states, and a transition rate $\Gamma_{\mathrm{ES}_{0,1} \rightarrow A_{1}}$ between the triplet excited states and the singlet manifold. We also include a transition rate $\Gamma_{A_{1}}$ for the decay from the singlet state to the $\mathrm{GS}_{0}$ and $\mathrm{GS}_{1}$ states where the so-called "branching ratio" $\mathcal{P}_{A_{1} \rightarrow \mathrm{GS}_{1}}$ describes the probability of decay into $\mathrm{GS}_{1}$ (with $1-\mathcal{P}_{A_{1} \rightarrow \mathrm{GS}_{1}}$ describing the probability of decay into $\mathrm{GS}_{0}$ ). Finally, we include the transition rate $\Gamma_{\text {ion }}$ from the $\mathrm{NV}^{-}$excited states $\left(\mathrm{ES}_{0}\right.$ and $\left.\mathrm{ES}_{1}\right)$ to the $\mathrm{NV}^{0}$ ground state $\left(\mathrm{GS}_{\mathrm{NV}^{0}}\right)$ and the transition rate $\Gamma_{\text {rec }}$ from the $\mathrm{NV}^{0}$ excited state $\left(\mathrm{ES}_{\mathrm{NV}^{0}}\right)$ to $\mathrm{NV}^{-}$ground states $\left(\mathrm{GS}_{1}\right.$ and $\left.\mathrm{GS}_{0}\right)$. We assume that both $\mathrm{ES}_{0}$ and $\mathrm{ES}_{1}$ ionize at the same rate $\Gamma_{\text {ion }}$ and that recombination

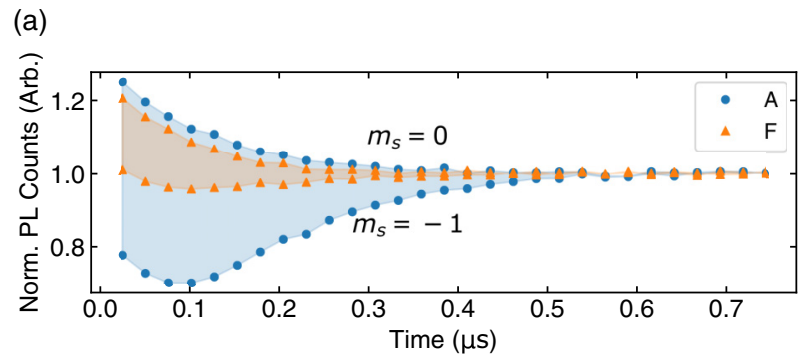

(b)

(c)
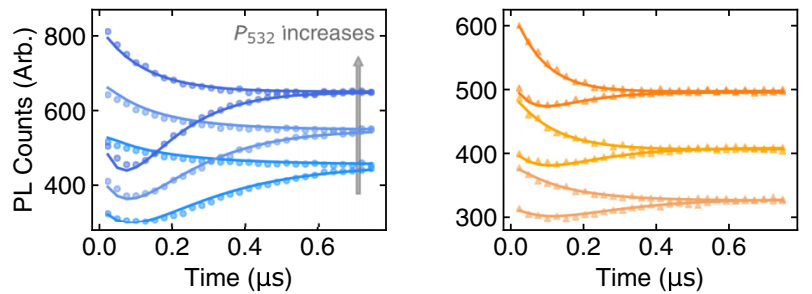

(d)

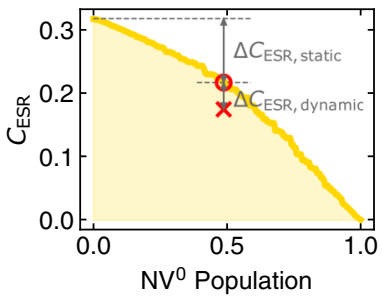

(e)

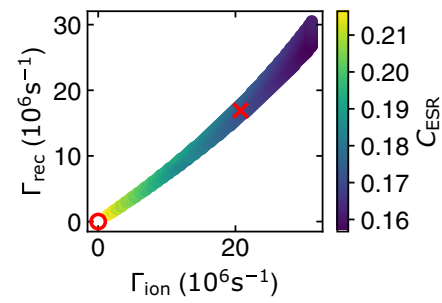

FIG. 5. Time-resolved PL traces and fitting to rate equation model. (a) Comparison of PL traces for representative NV centers from the two samples, A (blue) and $\mathrm{F}$ (orange), under $664 \mu \mathrm{W}$ of green illumination. PL counts are normalized to steady-state values for each trace. The shaded area is proportional to the OD-ESR contrast. (b), (c) Power dependence of NV PL traces from sample A (b) and sample $\mathrm{F}$ (c) after initialization into the $\mathrm{NV}^{-} m_{s}=0$ state and $m_{s}=-1$ state. Each trace represents a different green illumination power (from bottom to top: $330 \mu \mathrm{W}, 450 \mu \mathrm{W}, 660 \mu \mathrm{W}$ ). Solid lines indicate fits to the data using the rate equation model. (d) Simulated contrast as a function of $\mathrm{NV}^{0}$ population using extracted parameters from the fit to the same NV as in (c), where the upper bound (dark yellow points) represents the highest attainable contrast for a particular $\mathrm{NV}^{0}$ population and the shaded region represents lower $C_{\text {ESR }}$ caused by faster charge state conversion. The red cross indicates the extracted $C_{\mathrm{ESR}}$ and $\mathrm{NV}^{0}$ population for this $\mathrm{NV}$ center, and the highest attainable contrast for this $\mathrm{NV}^{0}$ population is indicated by the red circle. The total decrease in contrast compared to $C_{\mathrm{ESR}}$ with no charge dynamics is parametrized by the change due to the finite $\mathrm{NV}^{0}$ population of $0.49, \Delta C_{\mathrm{ESR} \text {,static }}$, and the change due to charge conversion rates, $\Delta C_{\mathrm{ESR}, \text { dynamic }}$. (e) $C_{\mathrm{ESR}}$ as a function of $\Gamma_{\text {ion }}$ and $\Gamma_{\text {rec }}$ for a fixed $\mathrm{NV}^{0}$ population $(49 \% \pm 1 \%)$. The red cross indicates the extracted $\Gamma_{\text {ion }}$ and $\Gamma_{\text {rec }}$ and the red circle indicates the point of highest $C_{\mathrm{ESR}}$. Inclusion of $\mathrm{NV}^{0}$ populations within $2 \%$ of $49 \%$ allows for a visual depiction of how sensitive $C_{\mathrm{ESR}}$ is to $\Gamma_{\text {ion }}$ and $\Gamma_{\text {rec }}$, showing that $C_{\mathrm{ESR}}$ is most sensitive to small changes in $\Gamma_{\text {ion }}$ and $\Gamma_{\text {rec }}$ when the magnitude of the rates is small.

from the $\mathrm{NV}^{0}$ excited state goes into the three spin states of $\mathrm{NV}^{-}$with equal probabilities [36]. With these transition rates the dynamics of the system can be described by the following 
TABLE I. Sample ion implantation details.

\begin{tabular}{lccc}
\hline \hline Sample & Surface condition & Implantation dose $\left(\mathrm{cm}^{-2}\right)$ & Implantation energy (keV) \\
\hline A & Polished + pre-etched, ${ }^{12}$ C enriched & $5 \times 10^{8}$ & 3 \\
B & As-grown, ${ }^{12}$ C enriched & $1 \times 10^{9}$ & 1.5 \\
C & Polished + pre-etched & $5 \times 10^{8}$ & 3 \\
D & Polished + pre-etched & $1 \times 10^{9}$ & 3 \\
E & As-grown, ${ }^{12}$ C enriched & $3 \times 10^{9}$ & 3 \\
F & Polished + pre-etched & $1 \times 10^{9}$ & 1.5 \\
\hline \hline
\end{tabular}

coupled rate equations, which are visualized in Fig. 4:

$$
\begin{aligned}
& \dot{\rho}_{\mathrm{ES}_{1}}=-\left(\Gamma_{\mathrm{ES}}+\Gamma_{\mathrm{ES}_{1} \rightarrow A_{1}}+\Gamma_{\mathrm{ion}}\right) \rho_{\mathrm{ES}_{1}}+\Gamma_{532} \rho_{\mathrm{GS}_{1}}, \\
& \dot{\rho}_{\mathrm{ES}_{0}}=-\left(\Gamma_{\mathrm{ES}}+\Gamma_{\mathrm{ES}_{0} \rightarrow A_{1}}+\Gamma_{\mathrm{ion}}\right) \rho_{\mathrm{ES}_{0}}+\Gamma_{532} \rho_{\mathrm{GS}_{0}} \text {, } \\
& \dot{\rho}_{A_{1}}=\Gamma_{\mathrm{ES}_{1} \rightarrow A_{1}} \rho_{\mathrm{ES}_{1}}+\Gamma_{\mathrm{ES}_{0} \rightarrow A_{1}} \rho_{\mathrm{ES}_{0}}-\Gamma_{A_{1}} \rho_{A_{1}}, \\
& \dot{\rho}_{\mathrm{GS}_{1}}=\Gamma_{\mathrm{ES}} \rho_{\mathrm{ES}_{1}}+\mathcal{P}_{A_{1} \rightarrow \mathrm{GS}_{1}} \Gamma_{A_{1}} \rho_{A_{1}}-\Gamma_{532} \rho_{\mathrm{GS}_{1}} \\
& +\left(2 \Gamma_{\text {rec }} / 3\right) \rho_{\mathrm{ES}_{\mathrm{NV} 0}} \text {, } \\
& \dot{\rho}_{\mathrm{GS}_{0}}=\Gamma_{\mathrm{ES}} \rho_{\mathrm{ES}_{0}}+\left(1-\mathcal{P}_{A_{1} \rightarrow \mathrm{GS}_{1}}\right) \Gamma_{A_{1}} \rho_{A_{1}}-\Gamma_{532} \rho_{\mathrm{GS}_{0}} \\
& +\left(\Gamma_{\mathrm{rec}} / 3\right) \rho_{\mathrm{ES}_{\mathrm{NV} 0}} \text {, } \\
& \dot{\rho}_{\mathrm{ES}_{\mathrm{NV} 0}}=-\left(\Gamma_{\mathrm{rec}}+\Gamma_{\mathrm{ES}_{\mathrm{Nv}}}\right) \rho_{\mathrm{ES}_{\mathrm{NV} 0}}+\Gamma_{532, \mathrm{NV}^{0}} \rho_{\mathrm{GS}_{\mathrm{NV}} 0} \text {, } \\
& \dot{\rho}_{\mathrm{GS}_{\mathrm{NV} 0}}=\Gamma_{\text {ion }} \rho_{\mathrm{ES}_{1}}+\Gamma_{\text {ion }} \rho_{\mathrm{ES}_{0}} \\
& +\Gamma_{\mathrm{ES}_{\mathrm{Nv}} 0} \rho_{\mathrm{ES}_{\mathrm{Nv} 0}}-\Gamma_{532, \mathrm{NV}^{0}} \rho_{\mathrm{GS}_{\mathrm{NV}} 0} \text {. }
\end{aligned}
$$

We then utilize the fact that

$$
\operatorname{PL}(t) \propto \Gamma_{\mathrm{ES}}\left[\rho_{\mathrm{ES}_{1}}(t)+\rho_{\mathrm{ES}_{0}}(t)\right]+\Gamma_{\mathrm{ES}_{\mathrm{NV} 0}} \rho_{\mathrm{ES}_{\mathrm{NV} 0}}(t)
$$

to predict the PL traces and then this model can be used to fit the experimental data [see Appendix $\mathrm{C}$ for additional information about Eq. (4)].

In order to constrain the model, we measure PL traces for both $m_{s}=0$ and $m_{s}=-1$ initial states for eight different laser powers and fit all data sets simultaneously to a single model. We constrain the fit to incorporate the same intrinsic NV parameters (excited state lifetime, singlet state lifetime, etc.) across all powers, while allowing the photoinduced transition rates to be free parameters. Representative PL traces for a subset of these powers for an NV center from sample A are shown in Fig. 5(b), and corresponding data sets from sample
F are shown in Fig. 5(c). Our fitted intrinsic NV parameters are consistent with previously reported measurements [32,34]. We also find that extracted excited state and singlet lifetimes are consistent across NV centers. A summary of the extracted parameters for four NV centers in each sample using a rate equation model with ionization and recombination and without ionization and recombination can be found in Table II and III, respectively. Importantly, all $C_{\mathrm{ESR}}$ values shown in Fig. 5 and Tables II and III are calculated from the fit function so that we can accurately predict how $C_{\mathrm{ESR}}$ changes relative to $\Gamma_{\text {ion }}$ and $\Gamma_{\text {rec }}$. Given the high quality of our fits, we find good agreement between values of $C_{\mathrm{ESR}}$ calculated from our model and the raw data and that the difference in $C_{\mathrm{ESR}}$ is typically $<0.02$.

From the data it is clear that there are two different contributions of charge state dynamics to decreased $C_{\mathrm{ESR}}$ : the increased background due to the steady-state $\mathrm{NV}^{0}$ population, and charge conversion rates interfering with spin dynamics. Armed with a model that quantitatively accounts for both spin and charge dynamics of the NV center, we use this model to disentangle the relative contributions to $C_{\mathrm{ESR}}$ of these two effects. Using the fitted NV parameters, we vary $\Gamma_{\text {ion }}$ and $\Gamma_{\text {rec }}$ to simulate values of $C_{\mathrm{ESR}}$ and the $\mathrm{NV}^{0}$ population. Using the extracted parameters for the NV center in sample $\mathrm{F}$ shown in Fig. 5(c), we plot the maximum $C_{\mathrm{ESR}}$ as a function of steady-state $\mathrm{NV}^{0}$ population [Fig. 5(d)]. As expected, $C_{\mathrm{ESR}}$

\begin{tabular}{|c|c|c|c|c|c|c|c|c|c|c|c|c|c|}
\hline Sampl & $\mathrm{V} \mathrm{Nc}$ & $C_{\mathrm{ESR}}$ & $\mathrm{P}_{\mathrm{NV}^{0}}(\%)$ & $\Gamma_{532}$ & on $(\mathrm{MHz})$ & $\Gamma_{\text {rec }}(\mathrm{MHz})$ & $\Gamma_{\mathrm{ES}}(\mathrm{MHz})$ & $\Gamma_{\mathrm{ESNV}^{0}}(\mathrm{MHz})$ & $\mathrm{ES}_{0} \tau(\mathrm{ns})$ & $\mathrm{ES}_{1} \tau$ & $A_{1} \tau$ & $P_{A_{1}} \rightarrow$ & Cost \\
\hline A & 10 & 0.20 & 0.11 & 19.6 & 5.6 & 390.3 & 75 & 36 & 10 & 8 & 60 & 0.25 & $1.79 \times 10^{-5}$ \\
\hline $\mathrm{A}$ & 16 & 0.20 & 0.32 & 24.9 & 21.2 & 176.6 & 75 & 36 & 12 & 10 & 104 & 0.25 & $3.34 \times 10^{-5}$ \\
\hline A & 17 & 0.42 & 0.11 & 24.2 & 4.2 & 45.2 & 75 & 16 & 12 & 8 & 104 & 0.25 & $4.57 \times 10^{-5}$ \\
\hline A & 11 & 0.35 & 0.08 & 14.0 & 2.8 & 74.8 & 75 & 16 & 12 & 8 & 88 & 0.25 & $8.53 \times 10^{-5}$ \\
\hline $\mathrm{F}$ & 78 & 0.15 & 0.30 & 18.3 & 5.5 & 8.3 & 75 & 36 & 8 & 6 & 62 & 0.37 & $9.85 \times 10^{-6}$ \\
\hline $\mathrm{F}$ & 91 & 0.17 & 0.49 & 22.4 & 20.7 & 16.9 & 70 & 32 & 11 & 8 & 124 & 0.25 & $1.02 \times 10^{-5}$ \\
\hline $\mathrm{F}$ & 40 & 0.23 & 0.21 & 11.1 & 0.1 & 0.2 & 75 & 20 & 9 & 7 & 69 & 0.25 & $2.96 \times 10^{-5}$ \\
\hline $\mathrm{F}$ & 24 & 0.29 & 0.25 & 9.4 & 3.8 & 6.3 & 75 & 16 & 10 & 7 & 86 & 0.25 & $3.97 \times 10^{-5}$ \\
\hline
\end{tabular}
decreases monotonically as the $\mathrm{NV}^{0}$ population increases, and we define $\Delta C_{\mathrm{ESR}, \text { static }}$ to be the decrease in $C_{\mathrm{ESR}}$ due to the finite $\mathrm{NV}^{0}$ population.

Separately, as the overall $\Gamma_{\text {ion }}$ and $\Gamma_{\text {rec }}$ increase in magnitude, the charge conversion dynamics begin to compete with

TABLE II. Fitted parameters based on the rate equation model incorporating ionization and recombination. Listed $C_{\mathrm{ESR}}$ (extracted from fits of the model), $\mathcal{P}_{\mathrm{NV}}, \Gamma_{532}, \Gamma_{\text {ion }}$, and $\Gamma_{\text {rec }}$ are the values at the maximum $C_{\mathrm{ESR}}$ across $p_{532}$ (and resultant $\Gamma_{532}$ ). We also include effective lifetimes $\mathrm{ES}_{0} \tau=1 /\left(\Gamma_{\mathrm{ES}}+\Gamma_{\mathrm{ES}_{0} \rightarrow A_{1}}\right), \mathrm{ES}_{1} \tau=1 /\left(\Gamma_{\mathrm{ES}}+\Gamma_{\mathrm{ES}_{1} \rightarrow A_{1}}\right)$, and $A_{1} \tau=1 / \Gamma_{A_{1}}$. It is worth noting that the actual excited state lifetime should also include ionization and recombination but we have selected these definitions to compare to models without ionization and recombination. $\Gamma_{\mathrm{ES}}$ is constrained to be $<75 \mathrm{MHz}$ and we are saturating the bound in our fit procedure. 
TABLE III. Fitted parameters based on the rate equation model without ionization and recombination. Listed $C_{\mathrm{ESR}}$ is extracted from fits of the model with ionization and recombination to be consistent with the method used in Figs. 5(d) and 5(e).

\begin{tabular}{|c|c|c|c|c|c|c|c|c|c|}
\hline Sample & NV No. & $C_{\mathrm{ESR}}$ & $\Gamma_{532}(\mathrm{MHz})$ & $\Gamma_{\mathrm{ES}}(\mathrm{MHz})$ & $\mathrm{ES}_{0} \tau(\mathrm{ns})$ & $\mathrm{ES}_{1} \tau(\mathrm{ns})$ & $A_{1} \tau(\mathrm{ns})$ & $\mathcal{P}_{A_{1} \rightarrow \mathrm{GS}_{1}}$ & Cost \\
\hline A & 10 & 0.20 & 16.1 & 68 & 10 & 8 & 56 & 0.25 & $2.18 \times 10^{-5}$ \\
\hline A & 16 & 0.20 & 11.8 & 45 & 11 & 9 & 63 & 0.35 & $8.09 \times 10^{-5}$ \\
\hline A & 17 & 0.42 & 20.5 & 68 & 12 & 8 & 93 & 0.25 & $7.71 \times 10^{-5}$ \\
\hline A & 11 & 0.35 & 11.5 & 67 & 12 & 8 & 81 & 0.25 & $1.20 \times 10^{-4}$ \\
\hline $\mathrm{F}$ & 78 & 0.15 & 13.6 & 44 & 11 & 10 & 49 & 0.40 & $2.47 \times 10^{-5}$ \\
\hline $\mathrm{F}$ & 91 & 0.17 & 9.4 & 33 & 11 & 9 & 62 & 0.30 & $4.29 \times 10^{-5}$ \\
\hline $\mathrm{F}$ & 40 & 0.23 & 11.3 & 63 & 11 & 9 & 53 & 0.25 & $4.57 \times 10^{-5}$ \\
\hline $\mathrm{F}$ & 24 & 0.29 & 8.7 & 49 & 13 & 9 & 66 & 0.25 & $9.44 \times 10^{-5}$ \\
\hline
\end{tabular}

the spin dynamics, leading to an additional decrease in $C_{\mathrm{ESR}}$. We define this additional decrease at a given $\mathrm{NV}^{0}$ population as $\Delta C_{\mathrm{ESR}, \text { dynamic. }}$. We plot a contour of the simulated $C_{\mathrm{ESR}}$ as a function of the charge state conversion rates at a fixed $\mathrm{NV}^{0}$ population [Fig. 5(e)], and we also observe a monotonic decrease of $C_{\mathrm{ESR}}$ with increasing $\Gamma_{\text {ion }}$ and $\Gamma_{\text {rec }}$.

For this particular NV center in sample $\mathrm{F}$, where $C_{\mathrm{ESR}}=$ 0.174 , we would expect an improvement to $C_{\mathrm{ESR}}=0.317$ in the absence of any charge conversion, where $\Delta C_{\mathrm{ESR}, \text { static }}=$ 0.100 and $\Delta C_{\mathrm{ESR} \text {,dynamic }}=0.042$. By comparison, for the $\mathrm{NV}$ center from sample A shown in Fig. 5(b) with $C_{\mathrm{ESR}}=$ 0.420 , the absence of charge conversion would yield a much smaller improvement in $C_{\mathrm{ESR}}$ to $0.453, \Delta C_{\mathrm{ESR} \text {,static }}=0.018$, and $\Delta C_{\mathrm{ESR}, \text { dynamic }}=0.015$.

\section{CONCLUSION}

We have shown that samples with different surface conditions can exhibit drastically different charge state dynamics for shallow NV centers, and that charge state stability can influence OD-ESR contrast in two ways: First, a high steadystate population in $\mathrm{NV}^{0}$ will increase the PL background, and second, when the charge state conversion time is comparable to the NV spin polarization and readout time, ionization results in a loss of spin information and initialization, and consequently a decrease of $C_{\mathrm{ESR}}$. These effects can be drastically different for different diamond samples, as well as among NV centers in a given sample. These results point to the importance of surface preparation and engineering in utilizing NV centers for nanoscale sensing and magnetometry. Ongoing and future work includes establishing microscopic mechanisms for charge state instabilities, such as surface trap states and contaminants, as well as exploring optimal surface terminations [37-40] for stabilizing the negative charge state of shallow NV centers.

\section{ACKNOWLEDGMENTS}

We thank James J. Allred for help with sample processing, Trisha Madhavan for help with part of the data acquisition, Haimei Zhang for help implementing the charge state readout optical setup, and Nan Yao, Yao-Wen Yeh, and John Schreiber at the Princeton Imaging and Analysis Center for help with diamond surface characterization. We also thank Dolev Bluvstein, Bo Dwyer, and Shimon Kolkowitz for helpful discussions. This material is based on work supported by the US Department of Energy, Office of Science, Office of Basic Energy Sciences, under Award No. DE-SC0018978. This work was also supported by the NSF under the CAREER program (Grant No. DMR-1752047), and was partially supported by the DARPA DRINQS program (Agreement No. D18AC00015). M.F. was supported by an appointment to the Intelligence Community Postdoctoral Research Fellowship Program by Oak Ridge Institute for Science and Education (ORISE) through an interagency agreement between the US Department of Energy and the Office of the Director of National Intelligence (ODNI). L.V.H.R. acknowledges support from the Department of Defense through the National Defense Science and Engineering Graduate Fellowship Program.

\section{APPENDIX A: DIAMOND SAMPLE PREPARATION}

Most diamond samples in this work are prepared following the procedure outlined in Ref. [7]. For samples A, C, D, and $\mathrm{F}$, we started with electronic grade diamonds from Element Six which were scaife polished to a RMS roughness of less than $1 \mathrm{~nm}$. We then performed inductively coupled plasma reactive ion etching followed by high-temperature annealing at $1200{ }^{\circ} \mathrm{C}$ in vacuum to remove surface and subsurface damage. After annealing, samples were cleaned in a refluxing mixture of 1:1:1 concentrated sulfuric, nitric, and perchloric acids (triacid clean) to remove surface amorphous carbon formed during annealing. Samples B and E did not go through this polish and pre-etch process and so their surfaces were "asgrown" before implantation. Next, NV centers were formed by nitrogen ion implantation followed by annealing at $800^{\circ} \mathrm{C}$ in vacuum. Further details about implantation parameters for different samples are listed in Table I. Afterward, samples were triacid cleaned again. This was the last step performed for sample B. For samples A, C, D, E, and F we subsequently annealed them in an oxygen atmosphere at temperatures around $440-460{ }^{\circ} \mathrm{C}$ to create well-ordered oxygen-terminated diamond surfaces. After oxygen annealing, these samples were cleaned in piranha solution (1:2 mixture of hydrogen peroxide and concentrated sulfuric acid).

To compare the OD-ESR contrast for NV centers in sample $\mathrm{A}$ and $\mathrm{F}$, we provide histograms of $C_{\mathrm{ESR}}$ in Fig. 1(c). Here, we also include probability density functions and cumulative distribution functions of $C_{\mathrm{ESR}}$ from these two samples (Fig. 6), which could help visually parse the difference.

Due to poor OD-ESR contrast in sample F, we are unable to use proton NMR to measure the depths of individual NV 
(a)

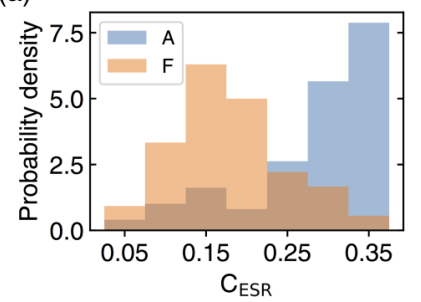

(b)

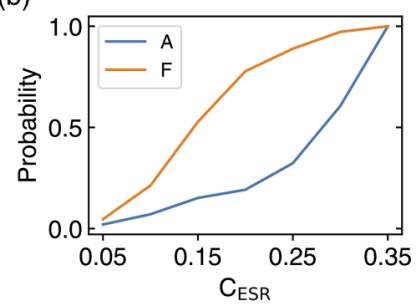

FIG. 6. Probability distribution functions for $C_{\mathrm{ESR}}$ from sample A (blue) and F (orange): (a) Probability density function and (b) cumulative distribution function. Data in Fig. 1(c) are used for calculating these probability distribution functions.

centers. Instead we provide a comparison sample that was implanted at the same energy, sample B. Histograms of the contrast and depth distributions for sample B are presented in Fig. 7. Although sample B was implanted with the same ion implantation energy as sample $\mathrm{F}$, we observe significantly higher OD-ESR contrast for NV centers in this sample, which suggests the lower contrast in sample F mainly results from the diamond surface condition.

X-ray photoelectron spectroscopy (XPS) characterization of the diamond surfaces was performed with a Thermo Fisher K-Alpha spectrometer at the Imaging and Analysis Center at Princeton University. XPS spectra for samples A, B, and F are shown in Fig. 8. A peak associated with boron $1 s$ is evident in sample F suggesting boron contamination on the surface, which has an atomic percentage of $0.31 \%$. We estimate that this atomic percentage corresponds to approximately $4 \%$ of a surface monolayer of boron using the method discussed in Ref. [7].

\section{APPENDIX B: EXPERIMENTAL SETUP}

NV photoluminescence (PL) measurements are performed in a home-built confocal microscope setup. A Nikon Plan Fluor $100 \times, \mathrm{NA}=1.30$, oil immersion objective is used for focusing the excitation laser and collecting the PL. For the green illumination, we use a $532 \mathrm{~nm}$ optically pumped solid-state laser (Coherent Sapphire LP 532-300), and for the orange illumination we use an NKT SuperK laser (repetition rate $78 \mathrm{MHz}$, pulse width $5 \mathrm{ps}$ ) with two bandpass filters with

(a)

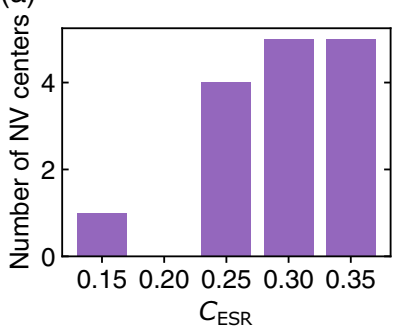

(a)

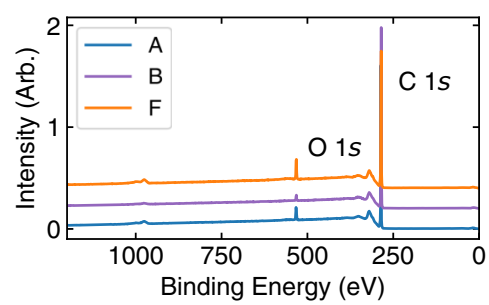

(b)

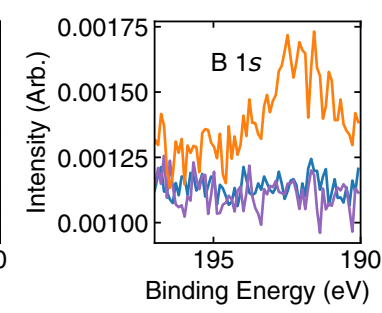

FIG. 8. XPS characterization of surfaces for samples A, B, and F. (a) XPS survey scans showing dominant carbon and oxygen peaks, shifted vertically for clarity. Samples A and F were annealed in an oxygen atmosphere and show higher oxygen $1 s$ peaks. (b) Highresolution XPS boron $1 s$ spectrum showing a small but clear peak in sample F. Intensities in both subplots are normalized by setting the peak height to 1 in the high-resolution carbon $1 s$ spectra for each sample.

transmission wavelength around $590 \mathrm{~nm}$ (Thorlabs FB590-10 and Semrock FF01-589/18-25). Both lasers are modulated by acousto-optic modulators (AOMs) (Isomet 1205C-1) and the beam is scanned with X-Y galvo mirrors (Thorlabs GVS012). A dichroic mirror (Semrock BLP01-647R-25) is used to separate the excitation and collection pathways, and the PL is measured using a fiber-coupled avalanche photodiode (Excelitas SPCM-AQRH-44-FC).

For the PL spectrum measurements shown in Fig. 2, we use a Princeton Instruments Monochromator (Acton SP-2300i) with a CCD camera (Pixis 100). Each spectrum is obtained by first acquiring the background signal of a non-NV spot close to the target NV and subtracting this background spectrum from the acquired NV spectrum. This technique allows us to remove background fluorescence and Raman lines of the driving laser.

All the pulse sequences used in the experiments are programmed by a PulseBlaster (SpinCore ESR-PRO500 with a timing resolution of $2 \mathrm{~ns}$ ). For time-resolved measurements of NV PL, the avalanche photodiode signal is sent to a PicoHarp (PicoQuant PicoHarp 300 with a highest resolution of 4 ps).

\section{APPENDIX C: TIME-RESOLVED PHOTOLUMINESCENCE MEASUREMENTS: DATA FITTING}

In our time-resolved measurements of NV PL, a green pulse is used to initialize the $\mathrm{NV}^{-}$spin state to $m_{s}=0$. Then we either leave the NV in $m_{s}=0$ or apply a calibrated microwave $\pi$ pulse to initialize the NV into $m_{s}=-1$. A subsequent green pulse is used to measure the PL which is read out by a PicoHarp to achieve high time resolution. We vary the power of both initialization and readout green pulses together to get the power dependence of the time-resolved PL. It is worth noting that this potentially changes the initial state that our model uses to predict the time-resolved PL. We address this in our modeling.

In order to quantify the optically induced spin dynamics of our NV centers in the presence of $\mathrm{NV}$ ionization and recombination, we expand on a model introduced in previous works [32-35]. A representation of the states of our system and the corresponding transition rates can be found in Fig. 4.

FIG. 7. Characteristics of NV centers in sample B, which was implanted at the same energy as sample $F$ but has an as-grown surface. (a) Histogram of OD-ESR contrast, $C_{\mathrm{ESR}}$. (b) Histogram of measured NV depths.

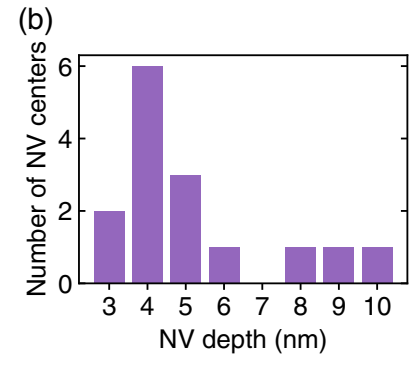

033263-7 
In our model, we use five levels to describe the $\mathrm{NV}^{-}$spin states, which include two states to describe the ground state triplet $\left(\mathrm{GS}_{0}\right.$ and $\left.\mathrm{GS}_{1}\right)$, two states from the excited state triplet $\left(\mathrm{ES}_{0}\right.$ and $\left.\mathrm{ES}_{1}\right)$, and a single state for the singlet manifold $\left(A_{1}\right)$. We also include ground and excited states for $\mathrm{NV}^{0}\left(\mathrm{GS}_{\mathrm{NV}^{0}}\right.$ and
$\left.\mathrm{ES}_{\mathrm{NV}^{0}}\right)$. This allows us to describe the system as a state vector [see Eq. (4)], which can be rewritten in a matrix form:

$$
\dot{\rho}_{\psi}(t)=\boldsymbol{R}_{M} \rho_{\psi}(t)
$$

$$
\boldsymbol{R}_{\boldsymbol{M}}=\left[\begin{array}{cc}
-\left(\Gamma_{\mathrm{ES}}+\Gamma_{\mathrm{ES}_{1} \rightarrow A_{1}}+\Gamma_{\text {ion }}\right) & 0 \\
0 & -\left(\Gamma_{\mathrm{ES}}+\Gamma_{\mathrm{ES}_{0} \rightarrow A_{1}}+\Gamma_{\text {ion }}\right) \\
\Gamma_{\mathrm{ES}_{1} \rightarrow A_{1}} & \Gamma_{\mathrm{ES}_{0} \rightarrow A_{1}} \\
\Gamma_{\mathrm{ES}} & 0 \\
0 & \Gamma_{\mathrm{ES}} \\
0 & 0 \\
\Gamma_{\text {ion }} & \Gamma_{\text {ion }}
\end{array}\right.
$$

For the readout, the NV center is initialized with the same green pulse. This means that the initial charge state can be estimated by solving for the steady state of the rate equation matrix $\left[\dot{\rho}_{\psi}(t)=0\right]$. The solution can be found as the zero eigenvector of $\boldsymbol{R}_{M}, \rho_{\psi, S S}$. We then sum the components of $\rho_{\psi, S S}$ from the $\mathrm{NV}^{0}$ manifold and the $\mathrm{NV}^{-}$manifold, which we call $\mathcal{P}_{\mathrm{NV}^{0}}$ and $\mathcal{P}_{\mathrm{NV}^{-}}$, respectively. Right before the readout, we assume that the NV is in the ground state because we have waited for a time longer than the excited state lifetimes. This means that if we initialize in $m_{s}=0$, then

$$
\rho_{\psi}(t=0)=\left[\begin{array}{c}
\rho_{\mathrm{ES}_{1}}(t=0) \\
\rho_{\mathrm{ES}_{0}}(t=0) \\
\rho_{A_{1}}(t=0) \\
\rho_{\mathrm{GS}_{1}}(t=0) \\
\rho_{\mathrm{GS}_{0}}(t=0) \\
\rho_{\mathrm{ES}_{\mathrm{NV}} 0}(t=0) \\
\rho_{\mathrm{GS}_{\mathrm{NV}}}(t=0)
\end{array}\right]=\left[\begin{array}{c}
0 \\
0 \\
0 \\
0 \\
\mathcal{P}_{\mathrm{NV}^{-}} \\
0 \\
\mathcal{P}_{\mathrm{NV}^{0}}
\end{array}\right],
$$

and if we initialize in $m_{s}=-1$, then

$$
\rho_{\psi}(t=0)=\left[\begin{array}{c}
\rho_{\mathrm{ES}_{1}}(t=0) \\
\rho_{\mathrm{ES}_{0}}(t=0) \\
\rho_{A_{1}}(t=0) \\
\rho_{\mathrm{GS}_{1}}(t=0) \\
\rho_{\mathrm{GS}_{0}}(t=0) \\
\rho_{\mathrm{ES}_{\mathrm{NV} 0}}(t=0) \\
\rho_{\mathrm{GS}_{\mathrm{NV} 0}}(t=0)
\end{array}\right]=\left[\begin{array}{c}
0 \\
0 \\
0 \\
\mathcal{P}_{\mathrm{NV}^{-}} \\
0 \\
0 \\
\mathcal{P}_{\mathrm{NV}^{0}}
\end{array}\right]
$$

These initial states are then evolved in time according to the rate equation matrix to give

$$
\rho_{\psi}(t)=e^{\boldsymbol{R}_{M} t} \boldsymbol{\rho}_{\psi}(t=0) .
$$

The PL measured in the experiment is predominantly from transitions from the excited state triplet to the ground state triplet of $\mathrm{NV}^{-}$(which occurs at a rate $\Gamma_{\mathrm{ES}}$ ) or from the excited state to ground state of $\mathrm{NV}^{0}$ (which occurs at a rate $\Gamma_{\mathrm{ES}_{\mathrm{NV} 0}}$ ). This means that given the model predictions for the population in each of the states as a function of time, we can calculate the PL using Eq. (4).

To obtain the actual PL, we need to take into account the total collection efficiency of our measurement setup. This will introduce an overall scale factor to Eq. (4) and convert the proportionality $(\propto)$ to an equality $(=)$. However, in order to reduce the number of fit parameters in our model, we normalize both the experimental data and the predicted PL from the model. It is worth noting that emissions from the

$\left.\begin{array}{ccccc}0 & \Gamma_{532} & 0 & 0 & 0 \\ 0 & 0 & \Gamma_{532} & 0 & 0 \\ -\Gamma_{A_{1}} & 0 & 0 & 0 & 0 \\ \mathcal{P}_{A_{1} \rightarrow \mathrm{GS}_{1}} \Gamma_{A_{1}} & -\Gamma_{532} & 0 & 2 \Gamma_{\mathrm{rec}} / 3 & 0 \\ \left(1-\mathcal{P}_{A_{1} \rightarrow \mathrm{GS}_{1}}\right) \Gamma_{A_{1}} & 0 & -\Gamma_{532} & \Gamma_{\mathrm{rec}} / 3 & 0 \\ 0 & 0 & 0 & -\Gamma_{\mathrm{rec}}-\Gamma_{\mathrm{ES}_{\mathrm{NV} 0}} & \Gamma_{532_{\mathrm{NV}}} \\ 0 & 0 & 0 & \Gamma_{\mathrm{ES}_{\mathrm{NV} 0}} & -\Gamma_{532_{\mathrm{NV}}}\end{array}\right]$.

$\mathrm{NV}^{-}$and $\mathrm{NV}^{0}$ charge states have different spectral distributions; the collection efficiency should be different and should be accounted for with an additional multiplicative factor in Eq. (4). However, it was found that in the limiting case where the $\mathrm{NV}^{0}$ charge state produces no PL, most of the parameters extracted from fitting the model are unchanged except for $\Gamma_{\text {rec }}$. However, since $\Gamma_{\text {rec }}$ is typically much faster than the other transition rates, this does not dramatically affect relevant quantities such as $C_{\mathrm{ESR}}$ and the $\mathrm{NV}^{0}$ population.

The PL traces of NV fluorescence were taken using the PicoHarp with a sampling resolution of 128 ps. Before fitting, the data are smoothed by averaging every 100 consecutive points. In order to remove the finite rise time of the AOM, we then search for the point after crossing our threshold where the instantaneous derivative of the PL changes from positive to negative, which we define as the $t=0$ point of the time trace. Each data set is then normalized such that the steady-state PL $=1$ (as described in the previous section). Then, we combine the data trace for the $m_{s}=0\left(\mathrm{GS}_{0}\right)$ initialization and the $m_{s}=-1\left(\mathrm{GS}_{1}\right)$ initialization for eight different laser powers $p_{532} \in\left[p_{0}, p_{1}, \ldots, p_{n}\right]$, where $n=8$. We then fit the set of sixteen curves with a single model that outputs all sixteen curves for a given set of parameters as a concatenated array. Fitting all the data sets in this way allows us to get a more precise measure of the NV parameters without overfitting.

To further avoid overfitting, we use a fit parameter $\beta_{532}$ which is multiplied by our measured laser power, $p_{532}$, to get $\Gamma_{532}$. Similarly, we expect the ionization and recombination rates to be roughly linear as a function of $p_{532}$. A linear scaling of $\Gamma_{\text {ion }}$ and $\Gamma_{\text {rec }}$ with $\Gamma_{532}$ yields the observed quadratic dependence of the charge state conversion rates as shown in Fig. 3. For NV centers in sample F there was a slight power dependence of the $\mathrm{NV}^{0}$ population and it generally decreased by a few percent across the full power range we measured. To account for this power dependence, we include additional parameters $\beta_{\text {ion,2 }}$ and $\beta_{\text {rec,2, }}$, which was multiplied by $p_{532}$ to give a slight quadratic scaling of $\Gamma_{\text {ion }}$ and $\Gamma_{\text {rec }}$. The net result was the following:

$$
\begin{gathered}
\Gamma_{532}\left(p_{532}\right)=\beta_{532} p_{532}, \\
\Gamma_{\text {ion }}\left(p_{532}\right)=\beta_{\text {ion }} p_{532}+\beta_{\text {ion }, 2} p_{532}^{2}, \\
\Gamma_{\text {rec }}\left(p_{532}\right)=\beta_{\text {rec }} p_{532}+\beta_{\text {rec }, 2} p_{532}^{2},
\end{gathered}
$$


where we have set the fit constraints such that $\beta_{\text {ion, } 2} \leqslant 0$ and $\beta_{\mathrm{rec}, 2} \geqslant 0$ so that the $\mathrm{NV}^{0}$ population is either constant as a function of $p_{532}$ or tends toward lower $\mathrm{NV}^{0}$ population as $p_{532}$ increases. To further reduce the number of free parameters in our model, we fix the excitation rate from the ground state to the excited state of $\mathrm{NV}^{0}$ as $\Gamma_{532, \mathrm{NV}^{0}} \equiv \Gamma_{532} / 3$ as is done in Ref. [32]. In the final model, the only free parameters are $\beta_{532}$, $\beta_{\text {ion }}, \beta_{\text {ion }, 2}, \beta_{\text {rec }}, \beta_{\text {rec }, 2}, \Gamma_{\mathrm{ES}}, \Gamma_{\mathrm{ES}_{\mathrm{Nv} 0}}, \Gamma_{\mathrm{ES}_{1} \rightarrow A_{1}}, \Gamma_{\mathrm{ES}_{0} \rightarrow A_{1}}, \Gamma_{A_{1}}$, and $\mathcal{P}_{A_{1} \rightarrow \mathrm{GS}}$. We also constrain $\Gamma_{\mathrm{ES}}<75 \mathrm{MHz}$ to produce physically realistic rates for $\Gamma_{\mathrm{ES}}$. We know that $\Gamma_{\mathrm{ES}}$ is determined by the optical density of states which we do not believe to be significantly different from other diamond samples in the literature [7,32-35]. It was found empirically that when $\Gamma_{\mathrm{ES}}$ is unconstrained, $\Gamma_{\mathrm{ES}}$ will increase dramatically to capture the time-domain PL which is believed to be due to charge dynamics and lead to a final fit which does not have physically relevant rates.

We then construct a simple cost function which computes the norm squared difference between the predicted and measured time-resolved PL and use this to quantify the quality of our fits. To avoid local minima in the fitting procedure, we run a full fit for a range of guesses of $\beta_{\text {ion }}$ and select the fit with the overall smallest cost function. This is done because it is believed that the cost function landscape is nonconvex and therefore is difficult to navigate by the gradient descent method. This is why we need to constrain parameters like $\Gamma_{\mathrm{ES}}$ in our fit (as described in the previous paragraph). Before considering the fit parameters of our full model, we fit the data to a simpler model that does not include $\mathrm{NV}^{0}$ as a comparison. The parameters extracted from the fits using the full model are shown in Table II and fits using a model which does not include ionization and recombination are shown in Table III. Results here suggest that adding ionization and recombination consistently improves the fit performance while producing intrinsic NV parameters that are consistent with the literature. The primary deviation is in the excited state lifetimes, which deviate from rates in Ref. [32] by at most a factor of two. It is also important to note that the values of $C_{\mathrm{ESR}}$ in both Table II and Table III are extracted from the fits of our model with ionization and recombination. This was done to be consistent with the analysis in Figs. 5(d) and 5(e) which extrapolated $C_{\mathrm{ESR}}$ from the fits. It was found that these values of $C_{\mathrm{ESR}}$ differ by $<4 \%$ from the values extracted from the raw PL traces.

\section{APPENDIX D: EFFECT OF IONIZATION AND RECOMBINATION ON CONTRAST AND SIGNAL-TO-NOISE RATIO}

Given that our model fits the experimental data well, we can see how $C_{\mathrm{ESR}}$ depends on various parameters in the model. In particular, we are interested in how $C_{\mathrm{ESR}}$ depends on $\Gamma_{\text {ion }}$ and $\Gamma_{\text {rec }}$. We begin by using the parameters which are extracted from fits of NV 17 in sample A and NV 91 in sample F. Then, we predict $C_{\mathrm{ESR}}$ based on these parameters but instead of using parameters $\beta_{\text {ion }}, \beta_{\text {ion, } 2}, \beta_{\text {rec }}, \beta_{\text {rec,2 }}$ we directly specify $\Gamma_{\text {ion }}$ and $\Gamma_{\text {rec }}$. Results of the expected $C_{\mathrm{ESR}}$, which includes optimizing the readout window to maximize $C_{\mathrm{ESR}}$, are plotted in Figs. 9(a) and 9(b) for NV 17 in sample A and NV 91 in sample F, respectively. These are the same NV centers used in Figs. 5(b) and 5(c)-5(e), respectively. The (a)

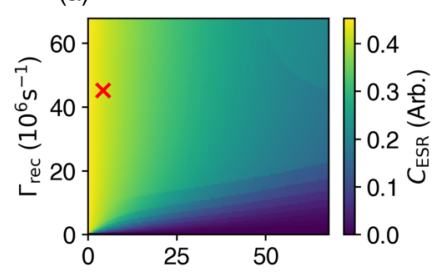

(c) $\Gamma_{\text {ion }}\left(10^{6} \mathrm{~s}^{-1}\right)$

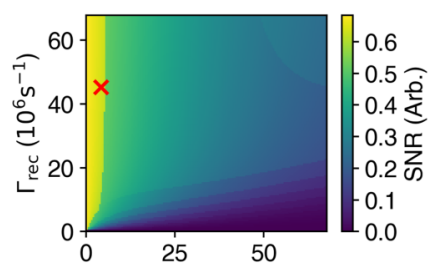

(e) $\Gamma_{\text {ion }}\left(10^{6} \mathrm{~s}^{-1}\right)$

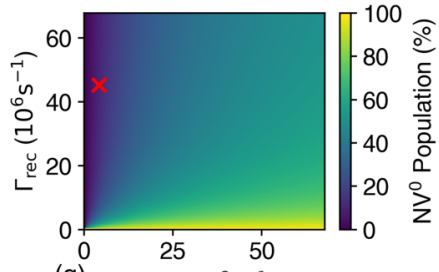

(g) $\Gamma_{\text {ion }}\left(10^{6} \mathrm{~s}^{-1}\right)$

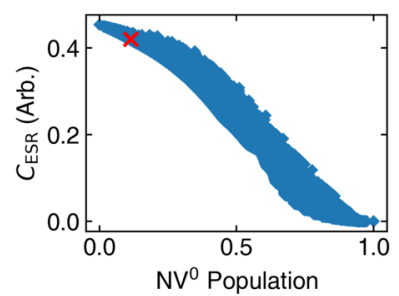

(b)

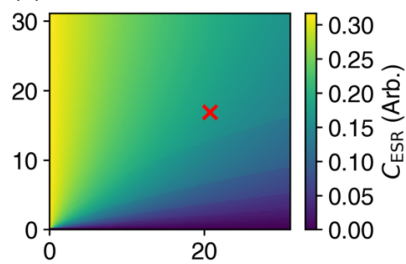

(d) $\quad \Gamma_{\text {ion }}\left(10^{6} \mathrm{~s}^{-1}\right)$

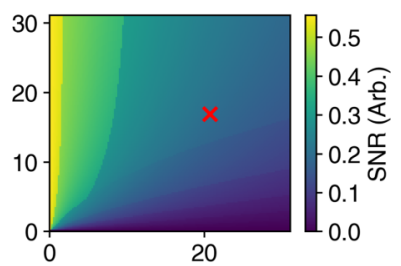

(f) $\Gamma_{\text {ion }}\left(10^{6} \mathrm{~s}^{-1}\right)$

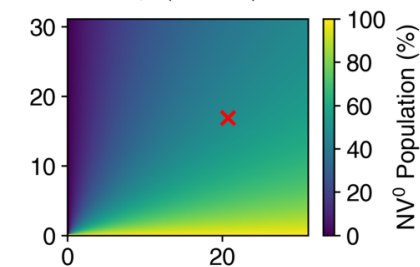

(h) $\quad \Gamma_{\text {ion }}\left(10^{6} \mathrm{~s}^{-1}\right)$

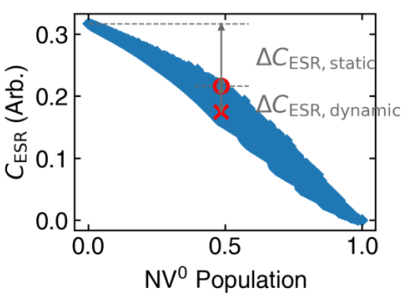

FIG. 9. (a), (b) Predicted OD-ESR contrast $C_{\mathrm{ESR}}$ as a function of $\Gamma_{\text {ion }}$ and $\Gamma_{\text {rec }}$ using the extracted parameters from representative NV centers from Fig. 5 from sample A and sample F, respectively. (c), (d) Predicted SNR as a function of $\Gamma_{\text {ion }}$ and $\Gamma_{\text {rec }}$ for the same NV centers. (e), (f) Steady-state $\mathrm{NV}^{0}$ population based on $\Gamma_{\text {ion }}$ and $\Gamma_{\text {rec }}$ for the same NV centers. (g), (h) Collected points from data in (a) and (e) or (b) and (f) that show $C_{\mathrm{ESR}}$ as a function of the $\mathrm{NV}^{0}$ population. The finite lower bound of $C_{\mathrm{ESR}}$ is a result of sampling from a finite range of $\Gamma_{\text {ion }}$ and $\Gamma_{\text {rec }}$. Red crosses correspond to the fitted $\Gamma_{\text {ion }}$ and $\Gamma_{\text {rec }}$ for the two NV centers. Based on the experimental contrast and the extracted parameter values we calculate a change in contrast due to finite $\mathrm{NV}^{0}$ population, $\Delta C_{\mathrm{ESR} \text {,static }}$, and due to the magnitude of the ionization and recombination rates $\Delta C_{\mathrm{ESR} \text {,dynamic }}$.

corresponding signal-to-noise ratio (SNR) following Ref. [28] is plotted in Figs. 9(c) and 9(d). Red crosses correspond to the parameters from the fitted data which correspond to the power which maximizes $C_{\mathrm{ESR}}$. In addition, based on $\Gamma_{\text {ion }}$ and $\Gamma_{\text {rec }}$ we calculate the $\mathrm{NV}^{0}$ population which is shown in Figs. 9(e) and 9(f). Finally, we use all of the $C_{\mathrm{ESR}}$ values in Figs. 9(a) and 9(b) and plot them against the $\mathrm{NV}^{0}$ populations in Figs. 9(e) and $9(\mathrm{f})$ to produce a scatter plot of $C_{\mathrm{ESR}}$ [Figs. $9(\mathrm{~g})$ and $\left.9(\mathrm{~h})\right]$ as a function of $\mathrm{NV}^{0}$ population, where it is clear that as the $\mathrm{NV}^{0}$ population $\rightarrow 100 \%, C_{\mathrm{ESR}} \rightarrow 0$. A similar trend holds for SNR. In addition, using the values shown in Figs. 9(g) and 9(h) we can quantify the decrease of contrast due to ionization and recombination by looking at the set of contrasts for a given $\mathrm{NV}^{0}$ population, where we attribute a change in 


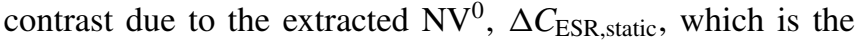
difference between $C_{\mathrm{ESR}}$ with all $\mathrm{NV}^{-}$population and the highest $C_{\mathrm{ESR}}$ at that $\mathrm{NV}^{-}$population. We can then attribute the change in contrast due to the magnitude of the ionization and recombination rates, $\Delta C_{\mathrm{ESR} \text {,dynamic }}$, which is the difference between the $C_{\mathrm{ESR}}$ of our NV center and the best $C_{\mathrm{ESR}}$ at the same $\mathrm{NV}^{0}$ population [see Figs. $9(\mathrm{~g})$ and $9(\mathrm{~h})$ for a visual representation of this]. It should be noted that $C_{\mathrm{ESR}}$ does not extend all the way to 0 because we have a finite upper bound for $\Gamma_{\text {ion }}$ and $\Gamma_{\text {rec }}$ in Figs. 9(a)-9(f).

\section{APPENDIX E: RELATIONSHIP BETWEEN $\Gamma_{\text {ion,rec }}$ and $r_{\text {ion,rec }}$}

To elucidate the relationship between $\Gamma_{\text {ion,rec }}$ and $r_{\text {ion,rec }}$ we consider a 4-level system, which consists of the ground state and excited state for $\mathrm{NV}^{-}\left(\mathrm{GS}_{\mathrm{NV}^{-}}\right.$: level 1 and $\mathrm{ES}_{\mathrm{NV}^{-}}$: level 2) and for $\mathrm{NV}^{0}\left(\mathrm{GS}_{\mathrm{NV}^{0}}\right.$ : level 3 and $\mathrm{ES}_{\mathrm{NV}^{0}}$ : level 4). We use $\rho_{i}$ to represent the population in level $i$ and from the conservation of total population we have $\sum_{i=1}^{4} \rho_{i}=1$.

We assume the conversion rate from level $i$ to level $j$ is $\Gamma_{i j}$. For each NV charge state we have photon-induced excitation rates $\Gamma_{12}, \Gamma_{34}$ and emission rates $\Gamma_{21}, \Gamma_{43}$. Also, we include the ionization rate from the $\mathrm{NV}^{-}$excited state to the $\mathrm{NV}^{0}$ ground state $\Gamma_{23}$ and the recombination rate from the $\mathrm{NV}^{0}$ excited state to the $\mathrm{NV}^{-}$ground state $\Gamma_{41}$.

The resultant rate equations are

$$
\begin{aligned}
& \dot{\rho}_{1}=-\Gamma_{12} \rho_{1}+\Gamma_{21} \rho_{2}+\Gamma_{41} \rho_{4}, \\
& \dot{\rho}_{2}=\Gamma_{12} \rho_{1}-\Gamma_{21} \rho_{2}-\Gamma_{23} \rho_{2}, \\
& \dot{\rho}_{3}=\Gamma_{23} \rho_{2}-\Gamma_{34} \rho_{3}+\Gamma_{43} \rho_{4}, \\
& \dot{\rho}_{4}=\Gamma_{34} \rho_{3}-\Gamma_{43} \rho_{4}-\Gamma_{41} \rho_{4},
\end{aligned}
$$

which can be rewritten as

$$
\dot{\rho}_{\psi, 4}(t)=\boldsymbol{R}_{M, 4} \rho_{\psi, 4}(t)
$$

where

$$
\boldsymbol{R}_{M, 4}=\left(\begin{array}{cccc}
-\Gamma_{12} & \Gamma_{21} & 0 & \Gamma_{41} \\
\Gamma_{12} & -\Gamma_{21}-\Gamma_{23} & 0 & 0 \\
0 & \Gamma_{23} & -\Gamma_{34} & \Gamma_{43} \\
0 & 0 & \Gamma_{34} & -\Gamma_{43}-\Gamma_{41}
\end{array}\right)
$$

and $\rho_{\psi, 4}=\left(\rho_{1}, \rho_{2}, \rho_{3}, \rho_{4}\right)^{T}$.

Starting from this full picture, we want to consider the conversion rates between the two charge states: $\rho_{\mathrm{NV}^{-}} \equiv \rho_{1}+$ $\rho_{2}$ and $\rho_{\mathrm{NV}^{0}} \equiv \rho_{3}+\rho_{4}$. Thus, the time derivative of the two charge state populations will be

$$
\begin{aligned}
\dot{\rho}_{\mathrm{NV}^{-}} & =-\Gamma_{23} \rho_{2}+\Gamma_{41} \rho_{4} \\
& =-\Gamma_{23} \frac{\rho_{2}}{\rho_{\mathrm{NV}^{-}}} \rho_{\mathrm{NV}^{-}}+\Gamma_{41} \frac{\rho_{4}}{\rho_{\mathrm{NV}^{0}}} \rho_{\mathrm{NV}^{0}}, \\
\dot{\rho}_{\mathrm{NV}^{0}} & =\Gamma_{23} \rho_{2}-\Gamma_{41} \rho_{4} \\
& =\Gamma_{23} \frac{\rho_{2}}{\rho_{\mathrm{NV}^{-}}} \rho_{\mathrm{NV}^{-}}-\Gamma_{41} \frac{\rho_{4}}{\rho_{\mathrm{NV}^{0}}} \rho_{\mathrm{NV}^{0}} .
\end{aligned}
$$

In general if the ratios $\rho_{2} / \rho_{\mathrm{NV}^{-}}$and $\rho_{4} / \rho_{\mathrm{NV}^{0}}$ change with time, we will not observe the exponential decay or increase in the populations. However, if the charge state conversion rates are much slower than the internal transition rates for each charge state, i.e., $\left\{\Gamma_{23}, \Gamma_{41}\right\} \ll\left\{\Gamma_{12}, \Gamma_{21}, \Gamma_{34}, \Gamma_{43}\right\}$, then on a timescale shorter than the charge state conversion timescale, the populations will reach a steady state within each charge state:

$$
\begin{aligned}
& \dot{\rho_{1}}=-\Gamma_{12} \rho_{1}+\Gamma_{21} \rho_{2}=0, \\
& \dot{\rho}_{2}=\Gamma_{12} \rho_{1}-\Gamma_{21} \rho_{2}=0, \\
& \dot{\rho}_{3}=-\Gamma_{34} \rho_{3}+\Gamma_{43} \rho_{4}=0, \\
& \dot{\rho_{4}}=\Gamma_{34} \rho_{3}-\Gamma_{43} \rho_{4}=0 .
\end{aligned}
$$

This leaves us with

$$
\frac{\rho_{2}}{\rho_{\mathrm{NV}^{-}}}=\frac{\Gamma_{12}}{\Gamma_{12}+\Gamma_{21}}, \quad \frac{\rho_{4}}{\rho_{\mathrm{NV}^{0}}}=\frac{\Gamma_{34}}{\Gamma_{34}+\Gamma_{43}},
$$

meaning that we can write the rate equation for two charge states as

$$
\begin{aligned}
& \dot{\rho}_{\mathrm{NV}^{-}}=-r_{\mathrm{ion}} \rho_{\mathrm{NV}^{-}}+r_{\mathrm{rec}} \rho_{\mathrm{NV}^{0}}, \\
& \dot{\rho}_{\mathrm{NV}^{0}}=r_{\mathrm{ion}} \rho_{\mathrm{NV}^{-}}-r_{\mathrm{rec}} \rho_{\mathrm{NV}^{0}},
\end{aligned}
$$

where $r_{\text {ion }}=\Gamma_{23} \Gamma_{12} /\left(\Gamma_{12}+\Gamma_{21}\right)$ and $r_{\text {rec }}=\Gamma_{41} \Gamma_{34} /\left(\Gamma_{43}+\right.$ $\left.\Gamma_{34}\right)$.

Now that we have derived effective rate equations for the populations in each of the charge states, we can explore how each of the individual transition rates affects the overall transition rate between charge states. The excitation from the ground state to the excited state, ionization, and recombination are each one-photon processes, which means that the rates $\Gamma_{12}, \Gamma_{34}, \Gamma_{23}$, and $\Gamma_{41}$ should each have linear power dependence. $\Gamma_{21}$ and $\Gamma_{43}$, which are the spontaneous emission rates analogous to $\Gamma_{\mathrm{ES}}$ and $\Gamma_{\mathrm{ES}}$, are decided by the intrinsic properties of NV centers and should not depend on power. If the laser power is much lower than the saturation power then $\Gamma_{12} \ll \Gamma_{21}$ and $\Gamma_{34} \ll \Gamma_{43}$. This means that $r_{\text {ion }}$ and $r_{\text {rec }}$ will each have a quadratic power dependence. If the laser power is much higher than the saturation power, then $\Gamma_{12} \gg \Gamma_{21}$ and $\Gamma_{34} \gg \Gamma_{43}$. This means that $r_{\text {ion }}$ and $r_{\text {rec }}$ should have linear power dependence, which agrees with the measurement results in Ref. [41]. This analysis demonstrates an intuitive relationship between individual transition rates in our rate equation model and $r_{\text {ion }}$ and $r_{\text {rec }}$ in various parameter regimes. It also qualitatively explains the power dependence of $r_{\text {ion }}$ and $r_{\text {rec }}$ in Fig. 3. It is worth noting, however, that the model here is a minimal model of the full rate equation model used in this paper, but we believe the same intuition holds for the full model albeit with slightly more cumbersome expressions.
[1] S. Hong, M. S. Grinolds, L. M. Pham, D. Le Sage, L. Luan, R. L. Walsworth, and A. Yacoby, MRS Bull. 38, 155 (2013).
[2] D. L. Sage, K. Arai, D. R. Glenn, S. J. Devience, L. M. Pham, M. D. Lukin, A. Yacoby, A. Komeili, and R. L. Walsworth, Nature (London) 496, 486 (2013). 
[3] P. C. Maurer, G. Kucsko, C. Latta, L. Jiang, N. Y. Yao, S. D. Bennett, F. Pastawski, D. Hunger, N. Chisholm, M. Markham, D. J. Twitchen, J. I. Cirac, and M. D. Lukin, Science 336, 1283 (2012).

[4] B. Hensen, H. Bernien, A. E. Dreaú, A. Reiserer, N. Kalb, M. S. Blok, J. Ruitenberg, R. F. L. Vermeulen, R. N. Schouten, C. Abellán, W. Amaya, V. Pruneri, M. W. Mitchell, M. Markham, D. J. Twitchen, D. Elkouss, S. Wehner, T. H. Taminiau, and R. Hanson, Nature (London) 526, 682 (2015).

[5] P. C. Humphreys, N. Kalb, J. P. J. Morits, R. N. Schouten, R. F. L. Vermeulen, D. J. Twitchen, M. Markham, and R. Hanson, Nature (London) 558, 268 (2018).

[6] I. Lovchinsky, A. O. Sushkov, E. Urbach, N. P. De Leon, S. Choi, K. De Greve, R. Evans, R. Gertner, E. Bersin, C. Muller, L. McGuinness, F. Jelezko, R. L. Walsworth, H. Park, and M. D. Lukin, Science 351, 836 (2016).

[7] S. Sangtawesin, B. L. Dwyer, S. Srinivasan, J. J. Allred, L. V. H. Rodgers, K. De Greve, A. Stacey, N. Dontschuk, K. M. O'Donnell, D. Hu, D. A. Evans, C. Jaye, D. A. Fischer, M. L. Markham, D. J. Twitchen, H. Park, M. D. Lukin, and N. P. De Leon, Phys. Rev. X 9, 031052 (2019).

[8] B. A. Myers, A. Das, M. C. Dartiailh, K. Ohno, D. D. Awschalom, and A. C. Bleszynski Jayich, Phys. Rev. Lett. 113, 027602 (2014).

[9] F. F. De Oliveira, D. Antonov, Y. Wang, P. Neumann, S. A. Momenzadeh, T. Häußermann, A. Pasquarelli, A. Denisenko, and J. Wrachtrup, Nat. Commun. 8, 15409 (2017).

[10] B. Grotz, J. Beck, P. Neumann, B. Naydenov, R. Reuter, F. Reinhard, F. Jelezko, J. Wrachtrup, D. Schweinfurth, B. Sarkar, and P. Hemmer, New J. Phys. 13, 055004 (2011).

[11] K. Ohno, F. Joseph Heremans, L. C. Bassett, B. A. Myers, D. M. Toyli, A. C. Bleszynski Jayich, C. J. Palmstrøm, and D. D. Awschalom, Appl. Phys. Lett. 101, 082413 (2012).

[12] B. Naydenov, F. Reinhard, A. Lämmle, V. Richter, R. Kalish, U. F. D'Haenens-Johansson, M. Newton, F. Jelezko, and J. Wrachtrup, Appl. Phys. Lett. 97, 242511 (2010).

[13] B. K. Ofori-Okai, S. Pezzagna, K. Chang, M. Loretz, R. Schirhagl, Y. Tao, B. A. Moores, K. Groot-Berning, J. Meijer, and C. L. Degen, Phys. Rev. B 86, 081406(R) (2012).

[14] M. Kim, H. J. Mamin, M. H. Sherwood, K. Ohno, D. D. Awschalom, and D. Rugar, Phys. Rev. Lett. 115, 087602 (2015).

[15] T. Rosskopf, A. Dussaux, K. Ohashi, M. Loretz, R. Schirhagl, H. Watanabe, S. Shikata, K. M. Itoh, and C. L. Degen, Phys. Rev. Lett. 112, 147602 (2014).

[16] Y. Romach, C. Müller, T. Unden, L. J. Rogers, T. Isoda, K. M. Itoh, M. Markham, A. Stacey, J. Meijer, S. Pezzagna, B. Naydenov, L. P. McGuinness, N. Bar-Gill, and F. Jelezko, Phys. Rev. Lett. 114, 017601 (2015).

[17] D. Bluvstein, Z. Zhang, and A. C. Bleszynski Jayich, Phys. Rev. Lett. 122, 076101 (2019).

[18] A. Stacey, N. Dontschuk, J.-P. Chou, D. A. Broadway, A. K. Schenk, M. J. Sear, J.-P. Tetienne, A. Hoffman, S. Prawer, C. I. Pakes, A. Tadich, N. P. de Leon, A. Gali, and L. C. L. Hollenberg, Adv. Mater. Interfaces 6, 1801449 (2019).
[19] S. Dhomkar, H. Jayakumar, P. R. Zangara, and C. A. Meriles, Nano Lett. 18, 4046 (2018).

[20] H. Yamano, S. Kawai, K. Kato, T. Kageura, M. Inaba, T. Okada, I. Higashimata, M. Haruyama, T. Tanii, K. Yamada et al., Jpn. J. Appl. Phys. 56, 04CK08 (2017).

[21] M. Pelliccione, A. Jenkins, P. Ovartchaiyapong, C. Reetz, E. Emmanouilidou, N. Ni, and A. C. B. Jayich, Nat. Nanotechnol. 11, 700 (2016).

[22] M. Batzer, B. Shields, E. Neu, C. Widmann, C. Giese, C. Nebel, and P. Maletinsky, Opt. Mater. Express 10, 492 (2020).

[23] P. Maletinsky, S. Hong, M. S. Grinolds, B. Hausmann, M. D. Lukin, R. L. Walsworth, M. Loncar, and A. Yacoby, Nat. Nanotechnol. 7, 320 (2012).

[24] P. Appel, E. Neu, M. Ganzhorn, A. Barfuss, M. Batzer, M. Gratz, A. Tschöpe, and P. Maletinsky, Rev. Sci. Instrum. 87, 063703 (2016).

[25] M. S. Grinolds, S. Hong, P. Maletinsky, L. Luan, M. D. Lukin, R. L. Walsworth, and A. Yacoby, Nat. Phys. 9, 215 (2013).

[26] M. Pelliccione, B. A. Myers, L. M. A. Pascal, A. Das, and A. C. Bleszynski Jayich, Phys. Rev. Appl. 2, 054014 (2014).

[27] D. A. Hopper, J. D. Lauigan, T.-Y. Huang, and L. C. Bassett, Phys. Rev. Appl. 13, 024016 (2020).

[28] D. A. Hopper, H. J. Shulevitz, and L. C. Bassett, Micromachines 9, 437 (2018).

[29] L. M. Pham, S. J. Devience, F. Casola, I. Lovchinsky, A. O. Sushkov, E. Bersin, J. Lee, E. Urbach, P. Cappellaro, H. Park, A. Yacoby, M. Lukin, and R. L. Walsworth, Phys. Rev. B 93, 045425 (2016).

[30] N. Aslam, G. Waldherr, P. Neumann, F. Jelezko, and J. Wrachtrup, New J. Phys. 15, 013064 (2013).

[31] S. T. Alsid, J. F. Barry, L. M. Pham, J. M. Schloss, M. F. O'Keeffe, P. Cappellaro, and D. A. Braje, Phys. Rev. Appl. 12, 044003 (2019).

[32] R. P. Roberts, M. L. Juan, and G. Molina-Terriza, Phys. Rev. B 99, 174307 (2019).

[33] L. Hacquebard and L. Childress, Phys. Rev. A 97, 063408 (2018).

[34] L. Robledo, H. Bernien, T. V. D. Sar, and R. Hanson, New J. Phys. 13, 025013 (2011).

[35] N. B. Manson, J. P. Harrison, and M. J. Sellars, Phys. Rev. B 74, 104303 (2006).

[36] X. D. Chen, L. M. Zhou, C. L. Zou, C. C. Li, Y. Dong, F. W. Sun, and G. C. Guo, Phys. Rev. B 92, 104301 (2015).

[37] M. Kaviani, P. Deák, B. Aradi, T. Frauenheim, J. P. Chou, and A. Gali, Nano Lett. 14, 4772 (2014).

[38] M. V. Hauf, B. Grotz, B. Naydenov, M. Dankerl, S. Pezzagna, J. Meijer, F. Jelezko, J. Wrachtrup, M. Stutzmann, F. Reinhard, and J. A. Garrido, Phys. Rev. B 83, 081304(R) (2011).

[39] A. Stacey, K. M. O'Donnell, J.-P. Chou, A. Schenk, A. Tadich, N. Dontschuk, J. Cervenka, C. Pakes, A. Gali, A. Hoffman, and S. Prawer, Adv. Mater. Interfaces 2, 1500079 (2015).

[40] S. Cui and E. L. Hu, Appl. Phys. Lett. 103, 051603 (2013).

[41] G. Waldherr, J. Beck, M. Steiner, P. Neumann, A. Gali, T. H. Frauenheim, F. Jelezko, and J. Wrachtrup, Phys. Rev. Lett. 106, 157601 (2011). 